Revue d'histoire de l'enfance « irrégulière »

Le Temps de l'histoire

5 | 2003

Pratiques éducatives et systèmes judiciaires

Les démarches législatives menant à la création des tribunaux pour mineur en Belgique, en France, aux Pays-Bas et au Québec au début du $\mathrm{XX}^{\mathrm{e}}$ siècle

Jean Trépanier

\title{
OpenEdition
}

Journals

Édition électronique

URL : http://journals.openedition.org/rhei/940

DOI : $10.4000 /$ rhei. 940

ISBN : 978-2-7535-1643-4

ISSN : $1777-540 \mathrm{X}$

Éditeur

Presses universitaires de Rennes

Édition imprimée

Date de publication : 15 novembre 2003

Pagination : 109-132

ISSN : 1287-2431

Référence électronique

Jean Trépanier, «Les démarches législatives menant à la création des tribunaux pour mineur en

Belgique, en France, aux Pays-Bas et au Québec au début du XX siècle ", Revue d'histoire de l'enfance " irrégulière » [En ligne], 5 | 2003, mis en ligne le 02 juin 2007, consulté le 04 décembre 2020. URL

http://journals.openedition.org/rhei/940 ; DOI : https://doi.org/10.4000/rhei.940 


\section{Les démarches législatives menant à la création des tribunaux pour mineurs en Belgique, en France, aux Pays-Bas et au Québec au début du XXème siècle}

C'est en évoquant la fondation du tribunal des mineurs de Chicago en 1899 que l'on a maintes fois déclaré centenaire la justice des mineurs en 1999. Il y a là une affirmation que d'aucuns contestent. Est-ce que, par exemple, l'expérience menée en Australie du Sud dès 1890 ne constitue pas le premier tribunal pour mineurs ? Les Child Welfare Boards institués par une loi norvégienne de 1896 doivent-ils se voir refuser le titre parce qu'ils étaient des organes administratifs plutôt que judiciaires? Aux États-Unis mêmes, quel poids doit-on accorder au célèbre juge et réformateur Ben Lindsey, selon qui le premier tribunal des mineurs aurait vu le jour chez lui, au Colorado, en 1899 ? Et que penser de l'affirmation du Canadien J. J. Kelso, surintendant des enfants négligés de l'Ontario à la fin du siècle dernier, qui n'hésitait pas à déclarer que le tribunal des mineurs " avait eu son origine à Toronto et qu'il était dès lors une "entreprise canadienne" que s'étaient appropriés des travailleurs sociaux américains » ? ${ }^{(2)}$

Un examen plus détaillé de ces diverses expériences nous montrerait que des différences significatives les distinguaient les unes des autres. Dans la mesure où l'on estime qu'il fallait plus que la simple tenue d'audiences séparées pour parler de tribunaux pour mineurs, chacune d'elles ne se qualifierait pas au même degré pour mériter l'appellation. Il reste que, quel que soit le continent où l'on logerait l'ancêtre des tribunaux pour mineurs, on peut fixer à la toute fin du XIXème siècle les débuts de la justice des mineurs, quelle qu'en soit la date très précise. Et surtout, on peut situer dans les premières années du XXème siècle leur rapide développement. Mises à part les rares lois adoptées à la toute fin du XIXème siècle (comme celles de la Norvège en 1896 et de l'Illinois en

Jean Trépanier / p. 109 à 132 
Anthony M. Platt, The

Child Savers : The

Invention of Delinquency,

2ème éd., Chicago,

University of Chicago

Press, 1977, p. 9. L'œuvre

de Platt demeure par

ailleurs centrale pour la

compréhension de la

création du tribunal de

Chicago.

(3) Lamar T. Empey et

Mark C. Stafford,

American Delinquency :

Its Meaning and Construc-

tion, 3ème éd., Belmont,

CA, Wadsworth

Publishing Company, 1991, p. 59.

(4) La seconde partie du texte ainsi que les conclusions qui s'en dégagent ont été préparées à partir de travaux menés par Marie-Sylvie Dupont-Bouchat (université catholique de Louvain) pour la Belgique, Éric Pierre (université d'Angers) pour la France, Jeroen Dekker (université de
1899), les lois organisatrices de la justice des mineurs datent de cette époque. On peut penser notamment à celles de l'Angleterre, de l'Irlande du Nord et du Canada en 1908 ; à celle du Portugal en 1911 ; à celles de la Belgique, de la France et de la Suisse en 1912 ; à celle de la Hongrie en 1913 ; à celle de la Pologne en 1918 ; à celles des Pays-Bas et du Japon en 1922 ; à celle de l'Allemagne en 1923 ; à celle de la Grèce en 1927 ; ou encore à celle de l'Autriche en 1929. On ne doit pas oublier les ÉtatsUnis où, vingt ans après l'institution du tribunal de Chicago, tous les États sauf trois avaient adopté des lois du même ordre. ${ }^{(3)}$

Ces lois - et d'autres auxquelles on pourrait faire référence - ne sont évidemment pas toutes semblables. Chacune porte la marque de la tradition juridique du milieu où elle s'incarne, de l'influence de personnages et de groupes de pression locaux ou nationaux, de compromis politiques. Aussi est-il apparu utile que, dans une étude comparée menée par plusieurs chercheurs de quatre pays, nous examinions l'adoption, avant la première guerre, de ces lois qui fondèrent la justice des mineurs en France, en Belgique, aux Pays-Bas et au Canada. ${ }^{(4)}$ La question centrale qui a orienté notre regard fut celle de savoir s'il n'y avait que coïncidence dans l'émergence de ces lois dans l'espace de quelques années seulement ou si une convergence pouvait être observée. Cette question est ici abordée en deux temps. De manière à mieux saisir le contexte dans lequel survint l'adoption de ces lois, un mot - même bref - s'impose sur les régimes juridiques qui visaient les mineurs avant l'adoption des lois qui nous intéressent ici, incluant le modèle de protection de l'enfance adopté par les Américains et qui servit d'inspiration à nombre de législateurs. Il sera ensuite possible d'examiner l'adoption des lois ellesmêmes dans les quatre pays concernés, pour conclure sur la question de savoir dans quelle mesure les lois elles-mêmes et les processus ayant mené à leur adoption dans les quatre pays étaient en convergence.

\section{Des statuts juridiques en développement}

Si elles innovent, les lois créant les tribunaux pour mineurs ne sont pas les premières qui conferent aux mineurs un statut particulier. Celuici commence à s'édifier graduellement au cours du XIXème siècle. On 
en a des indices certains dans les pays qui nous intéressent ici. On peut le voir également aux États-Unis, où se développe un modèle de protection de l'enfance qui aura une influence déterminante sur les tribunaux pour mineurs.

\subsection{Quelques indicateurs en Europe et au Canada}

Outre la mise en place d'institutions réservées aux mineurs, divers indices témoignent de ce qu'un statut particulier à ceux-ci s'établit par couches successives dans divers pays. On le voit notamment en France, où le Code pénal de 1810 introduit pour le mineur qui a agi sans discernement, l'acquittement accompagné d'une mesure " propre à rectifier son éducation $" .{ }^{(5)}$ Le mineur discernant, quant à lui, bénéficie de l'excuse atténuante de minorité, ce qui lui vaut des peines moindres qu'un adulte. Ce régime sera étendu à d'autres pays d'Europe continentale où se fait sentir l'influence française, et notamment la Belgique et les Pays-Bas.

On le voit encore dans des pays de common law qui adoptent des lois favorisant le recours à des procédures sommaires (ce qui vise à réduire la détention provisoire en compagnie d'adultes) et qui réduisent les peines applicables dans certains cas d'infractions relativement peu graves. Dans la foulée de l'Angleterre qui avait opté pour cette voie à compter de 1847, le Canada s'y engage à compter de 1857.

Certains législateurs vont même jusqu'à prévoir que les affaires impliquant des mineurs soient entendues séparément de celles qui concernent des adultes. À l'instar de certains États américains, ${ }^{\left({ }^{(}\right)}$le Canada insère dans son premier Code criminel (1892) des dispositions permettant la tenue de procès séparés de ceux des adultes. En France, le même objectif est visé non par une loi, mais par une circulaire de la Chancellerie en 1898. ${ }^{(7)}$ Ainsi des villes comme Paris et Toronto voient se mettre en place des pratiques par lesquelles les mineurs sont jugés lors d'audiences séparées de celles des adultes. De même, malgré l'absence d'assises légales, on met en place le recours à la liberté surveillée ; cela se fait dans certaines villes françaises, par l'intermédiaire du patronage de l'enfance et de l'adolescence, et dans des villes canadiennes comme Ottawa ou Montréal, grâce à l'intervention de sociétés d'aide à l'enfance.
Groningen) pour les

Pays-Bas et Jean Trépanier (université de

Montréal) pour le Canada. Tout le crédit doit être rendu à ces chercheurs. Les résultats de ces travaux ont fait l'objet d'un chapitre dans un rapport (1995) et un ouvrage : Marie-Sylvie Dupont-Bouchat et Éric Pierre, [dir.], Enfance et justice au XIXème siècle. Essais d'histoire comparée de la protection de l'enfance, 1820-1914. France, Belgique, Pays-Bas, Canada, Paris, PUF, 2001, 443 p. Une partie du présent texte vise à faire état de ces travaux ; elle en est donc forcément inspirée.

(5) Philippe Robert, Traité de droit des mineurs, Paris, éditions Cujas, 1969.

(6) On pense particulièrement aux États du Massachusetts (1874) et de New York (1892). 
(7) Voir Philippe

Robert, op. cit., p. 78-79.

(8) L'affaire Ex parte

Crouse a été résumée par plusieurs auteurs. Voir notamment Marvin

Ventrell, « Evolution of the dependency component of the juvenile court ", Juvenile and Family Court Journal, n 49/4, 1998, p. 23. Steven L. Schlossman, Love and the American Delinquent : The Theory and Practice of "Progressive" Juvenile Justice, 1825-1920, Chicago, University of Chicago Press, 1977, p. 8-10 ; «Delinquent children : The juvenile reform school ", in Norval Morris et David J. Rothman (éd.), The Oxford History of the Prison: The Practice of Punishment in Western Society, Oxford, Oxford University Press, 1995, p. 366 ; Danielle Laberge, Marginaux et marginalité : Les États-
La fin du siècle voit également apparaittre des lois visant la protection de l'enfance en danger. En France, l'adoption de la loi du 24 juillet 1889 marque une brèche dans l'autorité paternelle auparavant jugée intouchable : certains parents qui mettent leurs enfants en danger pourront être déchus de la puissance paternelle. En Belgique, la même année, le ministre de la Justice, Jules Lejeune, introduit une proposition de loi qui vise le même objectif. Si l'opposition rencontrée par le projet n'en permet pas l'adoption rapide, Lejeune fait néanmoins procéder à l'adoption d'autres dispositions visant la protection de l'enfance. Quant à la déchéance de la puissance paternelle, elle sera finalement intégrée à la loi de 1912.

Au Canada, si la constitution attribue au Parlement fédéral la compétence en matière de délinquance des mineurs, c'est aux provinces qu'est réservé le domaine de l'enfance en danger. Le Québec légifère en 1869 pour établir des "écoles d'industrie" où peuvent être placés les enfants négligés et abandonnés, alors que l'Ontario crée à compter de 1893 des sociétés d'aide à l'enfance dont le mandat est d'intervenir auprès des enfants en danger et de prévenir dans la mesure du possible leur placement en institution.

$\mathrm{Si}$ incomplets et schématiques soient-ils, ces quelques repères permettent de voir que, au début du XXème siècle, un statut juridique distinct avait déjà été mis en place pour les mineurs, et ce, en sus de l'établissement d'institutions qui leur soient réservées pour fins de détention et de placement. Les lois reconnaissaient déjà que les mineurs devaient être traités différemment des adultes. Qui plus est, un régime de protection de l'enfance commençait à se mettre en place. C'est à partir de ces assises que, dans chaque pays, pourrait cheminer le processus législatif qui mènerait à l'adoption des lois qui établiraient les tribunaux pour mineurs. Mais pour cela, un modèle importé des États-Unis contribuerait à inspirer les législateurs.

\subsection{Les États-Unis et le modèle de protection de l'enfance}

On associe fréquemment - et à juste titre - le développement des tribunaux pour mineurs aux États-Unis à une doctrine juridique, dite parens patriae, qui fut développée par les tribunaux américains à comp- 
ter des années 1830. L'affaire Crouse, ${ }^{(8)}$ qui remonte à 1839, est à son origine. Dans cette affaire, Mary Ann Crouse avait été confiée à la maison de refuge de Philadelphie à la demande de sa mère, mais hors de la connaissance de son père. Sa mère alléguait qu'elle était incontrôlable et que le placement était dans le meilleur intérêt de son enfant. Une fois mis au courant de la situation, le père de Mary Ann s'adressa aux tribunaux pour contester la décision, invoquant que l'incarcération de sa fille sans procès par jury violait la Constitution américaine. La requête du père fut rejetée par le tribunal qui se fonda sur une ancienne doctrine juridique anglaise, la doctrine parens patriae, qui permettait au monarque d'intervenir pour défendre les intérêts d'un mineur non représenté devant la Cour de chancellerie. Le tribunal estima que l'enfant avait été placée pour sa réforme, non dans un objectif de punition, ce qui justifiait que l'intervention de l'État soit vue comme étant dans l'intérêt de l'enfant ; dès lors, le tribunal pouvait ordonner le placement sans suivre les règles de procédure applicables en matière criminelle, la procédure étant définie comme une procédure civile, ce qui écartait les garanties procédurales offertes aux accusés par la Constitution américaine. Une série de décisions rendues dans les décennies suivantes vinrent confirmer cette approche, qui se retrouverait plus tard au cœur même de l'orientation des tribunaux pour mineurs.

Il suffit d'examiner la loi de l'Illinois qui crée le célèbre tribunal des mineurs de Chicago en 1899 pour se convaincre de cette filiation. Cette loi, qui est typique des lois américaines adoptées à cette période, ${ }^{(9)}$ se situe tout à fait dans la lignée de la doctrine parens patriae. Son dernier article le laisse bien voir :

"Cette loi sera interprétée libéralement, de manière à réaliser son objectif, à savoir que le soin, la garde et la discipline d'un enfant se rapprocheront autant que possible de ceux que devraient lui donner ses parents $[\ldots]{ }^{(10)}$

La procédure judiciaire aussi bien que les mesures imposées par le tribunal doivent refléter cette approche paternelle (si ce n'est maternelle, comme certains l'ont plutôt suggéré).

Contrairement à l'École classique et à l'idéologie libérale qui avaient
Unis aux XVIIème et

XIXème siècles, Paris,

L'Harmattan, 1997,

p. $137-139$; Sandford

J. Fox, «Juvenile justice

reform : An historical

perspective ", Stanford

Law Review, $\mathrm{n}^{\circ} 22$,

p. $1205-1207$.

(9) Aux États-Unis, les lois relatives aux tribunaux pour mineurs relèvent de la compétence des États. Même si les lois adoptées par les différents États à cette époque partageaient la même orientation, elles n'étaient pas en tout point semblables.

(10) « This act shall be liberally construed, to the end that its purpose may be carried out, towit : That the care, custody and discipline of a child shall approximate as nearly as may be that which should be given by its parents [...] " 
(11) Dominique

Dessertine et Bernard

Maradan, Pratiques judiciaires de l'assistance éducative (1889-1941), Lyon, Centre Pierre Léon, université Lumière-Lyon 2, 1991, p. 2. dominé le XIXème siècle, le comportement délinquant des enfants n'est plus vu à l'époque comme le fruit libre et délibéré de décisions rationnelles. Dans la foulée de l'École positiviste, on a plutôt tendance à l'associer à des facteurs extérieurs au délinquant, sur lesquels celui-ci n'a pas de contrôle. On pense notamment à des facteurs génétiques ou à l'environnement familial et social. Si l'infraction résulte de facteurs que le délinquant ne peut contrôler, une punition peut-elle être légitime ? Peut-on punir le délinquant en raison de ce que son environnement a fait de lui ? La réponse est évidemment négative. Le délinquant doit plutôt être protégé contre ces facteurs qui le mènent à la délinquance : c'est ainsi que la société sera le mieux protégée. La protection de l'enfance n'est pas vue comme s'opposant à la protection de la société. Bien au contraire, elle apparait comme le meilleur moyen de la réaliser. La distinction entre l'enfant délinquant et l'enfant en danger s'estompe, le second étant souvent perçu comme un délinquant en puissance. «À travers l'enfance malheureuse ou maltraitée, ce sont les éléments de "l'armée du crime" que l'on aperçoit et qui hantent le discours philanthropique, puis criminologique » de la fin du XIXème siècle et du début du XXème siècle. ${ }^{(1)}$ On estime qu'une approche de protection de l'enfance doit être privilégiée pour les deux groupes qui, dans les lois de certains États, seront fusionnés en un seul.

Ce modèle de protection - souvent désigné sous l'appellation de modèle welfare - se reflète de diverses manières et à divers degrés dans les lois adoptées au début du siècle. On peut toutefois identifier sa présence d'après les caractéristiques suivantes. La détention provisoire doit se faire dans des lieux où les adultes ne sont pas détenus : les jeunes doivent être protégés du danger de contamination que présentent les délinquants adultes. Le principe de proportionnalité et les mesures à durée déterminée doivent être abandonnés. Comme il vise à punir, le droit criminel classique requiert que la nature et la durée de la mesure pénale soient proportionnelles à la gravité de l'infraction, ce qui fait l'objet d'une décision au moment de l'imposition de la peine, avant son exécution. Par contre, si l'objectif de la mesure n'est plus de punir, mais de protéger l'enfant pour qu'il ne devienne pas délinquant, cette exi- 
gence perd tout son sens. Le choix de la mesure doit être centré sur les besoins individuels du mineur, non sur l'infraction. La mesure doit être appliquée aussi longtemps que le besoin s'en fait sentir. On peut la prolonger ou la modifier en cours d'exécution, selon l'évolution de ce dernier. Une fois le comportement délinquant ou la situation de danger établie devant le tribunal, le jeune peut demeurer pupille du tribunal ou de l'État jusqu'à sa majorité. Quant à la protection des droits par des garanties procédurales, elle apparait superflue, voire incompatible avec une approche centrée sur la protection de l'enfance : pourquoi reconnaîtrait-on à un enfant des droits qui lui permettraient de se protéger contre une intervention qui est vue comme lui étant favorable ? Ne serait-ce pas contraire à son intérêt ? C'est ainsi que l'on opte pour une procédure informelle, qui vise à favoriser une communication simple et directe entre le juge paternel et l'enfant, fût-ce au détriment des protections que le droit pénal reconnaît normalement aux accusés.

Cette orientation protectrice apparait toutefois trop contraire aux pratiques des tribunaux ordinaires pour qu'on puisse croire que ceux-ci la mettront vraiment en œuvre. Aussi faut-il prévoir la présence de deux nouveaux acteurs. Le juge des mineurs tout d'abord. Un juge paternel, bienveillant, protecteur, qui préside à une nouvelle juridiction spéciale, choisi en raison de ses qualités humaines et de son aptitude à comprendre les enfants. C'est un juge unique : le climat qui doit imprégner les rapports qu'il doit établir avec l'enfant serait incompatible avec le contexte par trop solennel et impressionnant que comporte un banc de plusieurs juges. Le second acteur, l'agent de probation ou délégué à la liberté surveillée, est le bras droit qui assiste ce magistrat spécialisé. Animé du même esprit que le magistrat, il mène ses interventions jusque dans la famille de l'enfant. Le modèle proposé par les tribunaux pour mineurs américains en est donc un qui mise sur la protection de l'enfance pour protéger la société.

Ce modèle sera présent dans les débats qui présideront à la conception et à l'adoption des lois dans bon nombre de pays, dont ceux qui nous intéressent ici. Sera-t-il pour autant adopté ? 


\section{L'adoption des lois canadienne, belge, française et néerlandaise}

Les lois permettant l'instauration de tribunaux pour mineurs furent adoptées successivement en 1908 au Canada, en 1912 en Belgique et en France, en 1922 aux Pays-Bas. Présentons brièvement dans l'ordre chronologique de leur adoption en quoi consistèrent ces lois et comment se caractérisa leur cheminement législatif, de manière à établir si une convergence se dégage d'un pays à l'autre.

\subsection{La loi canadienne sur les jeunes délinquants de $\mathbf{1 9 0 8}$}

Le processus même d'adoption de la loi canadienne sur les jeunes délinquants fait ressortir jusqu'à quel point cette loi fut influencée à la fois par les États-Unis et par l'orientation de réseaux canadiens liés à la protection de l'enfance. Le modèle protecteur y est tout à fait endossé.

Comme il a été déjà indiqué, la province d'Ontario avait exercé sa compétence législative en matière de protection de l'enfance en danger en 1893, lorsqu'elle avait adopté une loi sur la protection des enfants négligés. On y avait notamment prévu la création d'un réseau de sociétés d'aide à l'enfance, lequel essaima rapidement dans les villes principales de la province dans la dernière décennie du siècle. L'une d'elles avait rapidement été fondée à Ottawa, la capitale canadienne. Résolument orientées vers le placement en famille d'accueil plutôt que vers le placement institutionnel, ces sociétés préconisaient une approche de protection de l'enfance visant à laisser les enfants dans la collectivité plutôt que de les en retirer. Convaincues du succès de leur approche, elles en vinrent à souhaiter l'étendre aux enfants délinquants. Une assise légale leur manquait toutefois : détenant leurs pouvoirs d'une loi provinciale, elles ne pouvaient imposer leurs interventions aux mineurs délinquants et à leurs familles sans y être fondées par une loi fédérale.

En 1906, le président de la Société d'aide à l'enfance d'Ottawa, William L. Scott, accepte une invitation à participer à la National Conference of Charities and Corrections des États-Unis, à Philadelphie. Sceptique au départ, hésitant à se rendre à l'invitation, il cède aux pressions de ses collègues et se rend à la conférence. Il en revient convaincu : les tribunaux pour mineurs et la probation doivent être implantés au Canada. Deux dames 
sont immédiatement engagées comme agentes de probation par la Société d'aide à l'enfance d'Ottawa et sont envoyées à Philadelphie pour s'initier à leurs nouvelles responsabilités. Mais la nécessité d'une loi s'impose.

Avec l'aide de quelques conseillers dont certains sont associés au milieu des sociétés d'aide à l'enfance, il rédige un projet de loi en s'inspirant des lois de l'Illinois et du Colorado (qui régissaient les tribunaux de Chicago et de Denver), ainsi que de celle de la Pennsylvanie. À l'examen, ce projet de loi s'avère un miroir du modèle protecteur américain : on y crée des tribunaux pour mineurs délinquants où le juge paternel et bienveillant, assisté d'un agent de probation, doit agir dans une perspective de protection de l'enfance. ${ }^{(12)}$ Pour des raisons que l'espace ne permet pas d'expliciter ici, Scott ne parvient pas à convaincre le ministre de la Justice de prendre le projet de loi à son compte et de le présenter au Parlement au nom du gouvernement.

S’ensuit une série de manœuvres visant à amener le ministre à changer d'avis. Grâce aux liens que Scott a établis avec certains child savers américains, on fait venir à Ottawa certaines des figures les mieux connues des tribunaux pour mineurs américains : le juge Ben Lindsey de Denver et Madame Schoff, présidente du Conseil national des mères et responsable du tribunal des mineurs de Philadelphie. En plus de conférences publiques, on leur ménage des rencontres avec des parlementaires et des ministres, incluant le Premier ministre Laurier. Avec l'aide de sociétés d'aide à l'enfance (comme celle de Montréal), on organise des conférences et débats publics dans diverses villes du pays ; on y obtient la participation non seulement de personnes officiellement liées aux sociétés d'aide à l'enfance (comme J. J. Kelso ou Scott), mais aussi celle de personnes aussi en vue que le juge en chef de la Cour suprême du Canada et le gouverneur général du Canada. ${ }^{(13)}$ On fait signer des pétitions par de très nombreux citoyens, incluant des personnes de grande notoriété. Avec les contacts dont il dispose dans la capitale canadienne grâce à son père qui est sénateur et secrétaire d'État dans le gouvernement de Laurier, Scott fait intervenir les personnes les plus haut placées auprès du ministre de la Justice, qui refuse toujours d'endosser le projet de loi. On opte alors pour une autre stratégie. Le père de William Scott, le sénateur Scott, convient de présenter le pro-

(12) On n'y aborde toutefois pas la question de la déchéance de la puissance paternelle, qui est au cœur de la loi française de 1889 et de la loi belge de 1912 : ce domaine est réservé à la compétence des provinces, qui seules peuvent légiférer en matière de droit civil.

(13) Faisant alors partie de l'Empire britannique, le Canada avait (comme encore aujourd'hui) comme chef d'État le monarque britannique, lequel était représenté pour l'exercice des fonctions fédérales par un gouverneur général. 
jet de loi à titre personnel au Sénat en 1907, étant entendu avec le ministre de la Justice que le projet de loi serait débattu sans que le processus d'adoption ne soit mené à terme à cette même session parlementaire. Les promoteurs nourrissaient l'espoir que le ministre de la Justice se laisserait finalement fléchir et accepterait de présenter le projet de loi au nom du gouvernement à la session suivante. C'était trop espérer. Constatant à la session de 1908 que le ministre de la Justice ne change pas de position, il est décidé qu'une version remaniée du projet de loi serait déposée au Sénat par le sénateur Béique, grand avocat d'affaires et trésorier de la Société d'aide à l'enfance de Montréal fondée peu de temps auparavant. Une fois adopté par le Sénat, le projet de loi est finalement présenté à la Chambre basse par le ministre de la Justice, à qui le Premier ministre a finalement forcé la main devant l'importance des manifestations publiques. Le projet de loi est donc adopté en juillet 1908.

On voit donc que la loi canadienne est essentiellement d'initiative privée, adoptée littéralement contre le gré du ministre de la Justice, sous la pression d'une opinion publique que les promoteurs issus des milieux de la protection de l'enfance ont mobilisée. Parmi ceux-ci, une figure extraparlementaire se détache : celle de William Scott, dont l'appartenance à plusieurs réseaux joue un rôle clé dans l'adoption de la loi. En raison du statut de son père, les milieux politiques et judiciaires du plus haut niveau lui sont accessibles dans la capitale canadienne. Par sa position dans les sociétés d'aide à l'enfance ontariennes, il a été exposé aux idées qui y prévalent et peut s'en faire le relais dans les milieux politiques. Par les liens plus récents qu'il a établis avec les milieux de la protection de l'enfance américains, il est au fait des développements qui ont cours aux États-Unis et peut s'en faire le promoteur au Canada. C'est donc aux milieux de la protection de l'enfance qu'est due la conception et l'adoption de la loi : inspirés par le modèle protecteur américain, ces milieux souhaitent étendre aux mineurs délinquants une approche protectrice qu'ils pratiquaient déjà à l'endroit d'enfants en danger.

La nouvelle loi n'est que partiellement nouvelle : elle intègre des éléments présents dans des lois antérieures, en leur ajoutant toutefois des éléments nouveaux majeurs. Ceux-ci incluent notamment l'institution 
de tribunaux pour mineurs et de la probation, ainsi que l'adoption d'une approche protectrice à l'endroit des mineurs délinquants. En ce sens, la loi canadienne de 1908 est à la fois une loi synthèse et une loi complémentaire. Elle constitue également ce qu'on pourrait appeler une "loi clôture" : sauf pour des amendements mineurs qui n'en affectent nullement l'orientation, elle marque la fin des réformes législatives jusqu'à ce que des débats nouveaux reprennent soixante ans plus tard. Elle demeurera en vigueur pendant trois quarts de siècle et c'est véritablement sous son régime que s'édifiera la justice des mineurs au Canada.

\subsection{La loi belge sur la protection de l'enfance de $\mathbf{1 9 1 2}$}

En 1889, l'année même de l'adoption en France de la loi sur l'enfance moralement abandonnée, le ministre de la Justice, Jules Lejeune, dépose au Parlement belge un projet de loi qui vise notamment à introduire la déchéance de la puissance paternelle. C'est d'abord cette question qui soulève le plus d'opposition. Devant l'hostilité que soulève cette immixtion jugée intolérable de l'État dans le champ privé de la famille, Lejeune opte pour une double stratégie.

D’une part, il fait adopter par le Parlement une série de réformes partielles qui, si elles ne constituent pas une loi synthèse sur la protection de l'enfance, préparent néanmoins le terrain. On peut notamment penser à l'introduction des patronages, à la transformation des anciennes institutions pénitentiaires et écoles de réforme en écoles de bienfaisance, ou encore à la loi de 1891 sur le vagabondage et la mendicité qui touche les mineurs. Par ailleurs, un ensemble de moyens de pression est mis en place, auquel le ministre de la Justice apporte sa contribution. Coup sur coup, à quatre ans d'intervalle, Lejeune organise à Anvers trois congrès internationaux sur la protection de l'enfance $(1890,1894,1898)$. Il y invite les plus prestigieux de ses collègues étrangers, visant à promouvoir ses idées en amenant ces collègues à faire état des avantages obtenus à l'étranger (notamment en France) par l'introduction de changements tels que la déchéance de la puissance paternelle. Faisant de la Belgique la «capitale internationale du patronage ", ${ }^{(1)}$ il établit un nouveau réseau d'échanges internationaux qui sert d'appui à la promotion de ses vues.

(14) Marie-Sylvie

Dupont-Bouchat, Éric Pierre, [dir.], op. cit., p. 350 . 
(15) C. Campioni,

Enquête sur la criminalité infantile, Bruxelles, Commission royale des patronages, 1908.

(16) Comité de défense des enfants traduits en justice dans l'arrondissement de Bruxelles, 1906.
Dans la foulée des décisions adoptées par le congrès de 1890, on assiste en Belgique à la création de diverses associations de défense des enfants traduits en justice, alors que les comités de patronage sont réunis en 1894 dans une fédération nationale, la Commission royale des patronages. Principalement créées à l'initiative de juristes, d'avocats ou de juges de paix, ces associations constituent des groupes de pression qui organisent des réunions et des conférences, qui éditent des brochures, qui font circuler des pétitions, qui lancent des enquêtes sur l'enfance "moralement abandonnée". Leurs membres participent aux congrès internationaux, où ils côtoient leurs homologues étrangers et échangent sur leurs expériences, contribuant ainsi à la consolidation de réseaux mis en place grâce à l'initiative de Lejeune. Selon leur vocation respective, certaines associations s'attachent plus à réclamer la déchéance de la puissance paternelle, alors que d'autres mettent plus l'accent sur la création de tribunaux pour enfants. La Commission royale des patronages lance notamment, en 1907, une vaste enquête sur la criminalité infantile, qui débouchera sur des recommandations, notamment en matière de tribunaux pour enfants. ${ }^{(15)}$

Des appuis sont également fournis par certains milieux de juristes. Même en l'absence de loi habilitante, il arrive que des tribunaux rendent des décisions qui, dans la pratique, introduisent la déchéance de la puissance paternelle à l'endroit de "mauvais parents". On peut voir là l'expression d'un besoin signalé par les tribunaux à l'intention du législateur, l'invitant à mettre le droit en accord avec les besoins sociaux. Par ailleurs, dans des villes comme Bruxelles et Louvain, des comités de juristes encouragés par Lejeune - voire même présidés par lui - mettent en place de nouvelles pratiques spécifiques aux enfants traduits en justice, publiant même un Manuel de l'enfant traduit en justice ${ }^{(16)}$ pour assurer l'efficacité et la diffusion de leur expérience. Ils organisent des manifestations où est débattue la question des tribunaux pour enfants, avec référence aux pratiques instituées dans divers pays.

Des personnalités belges participent à des congrès internationaux où se discute la question des tribunaux pour mineurs. De tels congrès se tiennent à l'étranger (Washington, 1910) aussi bien qu'en Belgique (Liège, 1905 ; Anvers, 1911). Ces personnes se font le relais de ces débats en 
Belgique. Un exemple particulièrement connu est celui de l'épouse du ministre de la Justice Henri Carton de Wiart qui, après avoir accompagné son mari au congrès pénitentiaire international de Washington de 1910, se fait la promotrice de l'institution de tribunaux pour enfants et entreprend une série de conférences pour sensibiliser l'opinion belge.

L'ensemble de ces initiatives sont d'origine essentiellement privée, mais elles sont encouragées et soutenues (quand ce n'est lancées) par le ministre de la Justice, dont l'initiative favorise la création et la mise en place de réseaux d'influence et de pression qui préparent le terrain pour la reprise des débats parlementaires en 1911 et l'adoption de la loi en mai 1912.

Parrainée par le ministre de la Justice Carton de Wiart, la loi de 1912 reprend des questions qui étaient présentes dans le projet de 1889, mais elle ne s'y limite pas. Outre un chapitre qui institue la déchéance de la puissance paternelle et un autre qui traite des délits contre la moralité ou la faiblesse des enfants, elle institue le juge des enfants et la liberté surveillée. C'est d'ailleurs sur ce dernier point que la loi de 1912 se distingue le plus du projet de 1889. Au cours de ces vingt-trois ans, l'idée du tribunal pour enfants est apparue et a mûri. La loi intègre donc le juge unique et paternel, qui peut prendre à l'endroit des mineurs des mesures protectrices de garde, d'éducation et de préservation. Des mesures peuvent être prises non seulement à l'endroit des auteurs d'infractions, mais aussi à l'endroit de ceux qui «donnent par leur inconduite ou leur indiscipline de graves sujets de mécontentement à leurs parents » (article 14). Allant de la réprimande au placement, ces mesures incluent la liberté surveillée, dont l'exécution sera confiée aux sociétés protectrices de l'enfance. La loi endosse entièrement le modèle protecteur, qu'elle applique aux mineurs auteurs d'infractions.

À cette différence près qu'elle est promue par le gouvernement, l'adoption de la loi belge de 1912 marque donc des similitudes avec celle de la loi canadienne. Les résistances que rencontre au départ le projet de loi sont contrées par des interventions d'initiative privée, s'appuyant sur des réseaux d'influence auxquels le ministre de la Justice n'est pas toujours étranger et qui tentent de mobiliser l'opinion publique, surtout celle des milieux plus directement concernés. Faisant appel à l'expérience déve- 
loppée sur place, mais aussi à celle d'autres pays (et particulièrement des États-Unis), les promoteurs optent pour un modèle qui rejoint très largement celui que l'on a instauré outre-Atlantique, que ce soit aux ÉtatsUnis ou au Canada. Comme la loi canadienne, la loi belge de 1912 constitue à la fois une loi de synthèse et une loi complémentaire en ce qu'elle intègre des éléments présents dans des lois antérieures, tout en ajoutant des éléments nouveaux majeurs (notamment l'institution de tribunaux pour mineurs et de la probation). Elle marque elle aussi la fin des réformes législatives pour plusieurs décennies : elle demeurera en vigueur jusqu'à ce que la loi de 1965 vienne la remplacer et consolider encore plus ses assises protectrices.

\subsection{La loi française sur les tribunaux pour mineurs et la liberté surveillée de 1912}

Dans la première décennie du XXème siècle, la tenue d'audiences spéciales pour les mineurs apparaît dans les pratiques de certains tribunaux à Paris et dans certaines grandes villes de province. Des tentatives de mise en place de la liberté surveillée voient également le jour, par l'intermédiaire du patronage de l'enfance et de l'adolescence. C'est à partir de ces pratiques que des idées de réforme législative vont prendre naissance, dans un contexte où des philanthropes feront connaître le modèle des tribunaux pour mineurs américains.

Ils le feront dans le cadre de ce que l'on a appelé " une campagne de cinq années " pour évoquer cet ensemble de débats qui mèneront vers l'adoption de la loi de 1912. On fait remonter le début de ce mouvement à une conférence faite par Édouard Julhiet à Paris, au Musée social, en février 1906, dans laquelle Julhiet prône l'introduction en France de tribunaux pour mineurs inspirés du modèle américain. Prononcée devant de nombreux acteurs du champ de la protection de l'enfance, cette conférence a un retentissement considérable. Outre sa publication dans des revues, on en trouve des résumés dans la presse spécialisée et même dans les journaux nationaux.

L'idée se répand très rapidement, grâce notamment à la mobilisation du monde philanthropique parisien. C’est ce réseau qui animera les 
débats et mettra en avant l'idée des tribunaux pour enfants. Un acteur individuel jouera un rôle central : Henri Rollet, un bâtisseur qui, sa vie durant, participe à toutes les actions, tous les congrès nationaux et internationaux, à tous les débats sur la protection de l'enfance. Un acteur collectif assume également un rôle essentiel : le Comité de défense des enfants traduits en justice, qui groupe principalement des magistrats, des avocats et des juristes, mais aussi des publicistes et des hommes politiques. Ce comité se veut un organe de réflexion sur le fonctionnement de la justice des mineurs aussi bien que, à l'occasion, un groupement d'aide aux jeunes. Organisant des séances d'étude qui débouchent sur la formulation de recommandations adressées aux pouvoirs publics, il devient en fait un organe de réflexion et de pression. Compte tenu de la qualité de ses membres, tous situés à des postes importants dans la magistrature, dans l'administration ou au barreau, le comité parisien joue un rôle particulièrement actif et efficace.

En 1911, alors que l'adoption de la loi semble connaitre quelques difficultés au Parlement, un vaste congrès international est organisé à Paris pour stimuler le mouvement. Tout le monde de la philanthropie y est mobilisé et les orateurs viennent du monde entier. De multiples commissions donnent lieu à de nombreux exposés sur les tribunaux pour mineurs, notamment sur les expériences étrangères. Un large volume reprend l'ensemble des contributions. ${ }^{(17)}$ Cette manifestation de force et la légitimité qu'assure l'internationalisation du débat semblent contribuer à la reprise du débat au Parlement.

Car les débats parlementaires durent depuis un certain temps déjà. Deux propositions de loi partielles (les propositions Deschanel et Drelon) ont déjà été votées par la Chambre des représentants, en 1910. Une proposition synthèse est élaborée (la proposition Ferdinand-Dreyfus) et c'est elle qui, après avoir été amendée, deviendra loi. Il faut cependant souligner qu'un changement d'orientation se produit entre les premières propositions (tant parlementaires qu'extraparlementaires) et l'adoption de la loi. L'orientation de départ est fort inspirée du modèle américain : elle s'incarne dans un juge unique, paternel et bienveillant, assisté d'un délégué à la liberté surveillée, qui recourt à des mesures à caractère protecteur,
(17) Congrès international des tribunaux pour enfants, Actes du congrès, travaux préparatoires, comptes-rendus analytiques et sténographiques, Paris, A. Davy, 1912. 
convaincu qu'en protégeant l'enfant il protégera la société. On trouve cette orientation chez des défenseurs extraparlementaires du projet (tels Julhiet et divers acteurs des comités de défense des enfants traduits en justice) ; on la retrouve également chez les promoteurs de propositions de loi antérieures au sein du Parlement (par exemple Drelon). Déjà, le rapport de Ferdinand-Dreyfus marque un retrait par rapport à cette orientation. Mais les amendements des parlementaires accentuent encore l'écart. En fin de parcours, l'on ne saurait plus prétendre que les tribunaux pour enfants et adolescents pour lesquels opte la loi française sont conçus en fonction de l'application aux enfants délinquants d'un modèle de protection de l'enfance : la rupture avec le droit pénal n'est pas consommée, malgré l'opinion contraire de certains juristes de l'époque. L'opinion des tenants du modèle américain n'aura pas prévalu, malgré l'institution d'un régime spécifique aux mineurs et de la liberté surveillée.

On saurait difficilement résumer cette loi fort complexe en quelques lignes. Qu'il suffise de dire que, en plus d'instituer la liberté surveillée, elle prévoit des régimes distincts pour les mineurs de moins de treize ans et pour ceux de treize à dix-huit ans. La principale différence se situe au niveau du jugement : pour les seconds, on crée le tribunal pour enfants et adolescents, qui dans la majorité des cas n'est que le tribunal de première instance qui se réunit en audience spéciale pour les mineurs. On tient donc pour les adolescents des audiences séparées de celles des adultes, sans pour autant créer une juridiction spéciale avec le magistrat spécialisé que serait le juge des enfants. Quant aux mineurs de moins de treize ans, ils sont considérés comme irresponsables et, dès lors, ne sont pas sujets à la loi pénale. Si une fois l'instruction close on estime nécessaire qu'une mesure soit prise pour leur éducation, c'est la chambre du conseil du tribunal civil qui en aura la responsabilité.

Après débats, le Parlement rejette donc l'idée du juge des enfants, ce juge unique qui se spécialise dans les affaires des mineurs, qui siège seul en se faisant proche de l'enfant, dans un contexte qui mettrait plus l'accent sur l'intimité que sur la solennité. Le choix se porte plutôt sur le maintien de juridictions qui rendent des décisions collégiales et que la tradition française voit comme un rempart contre l'arbitraire du juge unique. 
Bref, en France comme ailleurs, l'adoption de la loi de 1912 est largement due à l'initiative privée. Des réseaux de personnes engagées à divers titres dans la protection de l'enfance s'organisent et recourent à des moyens de pression visant à mobiliser l'opinion, du moins celle d'un public éclairé. L'émergence de cette loi n'est pas étrangère aux pratiques qui ont été expérimentées en France dans les années antérieures. Les débats qui mènent à son adoption font largement état de modèles étrangers, et tout particulièrement du modèle américain. Mais contrairement à la Belgique et au Canada, la France ne retient pas ce dernier : c'est plutôt avec l'ordonnance de 1945 qu'apparaittra véritablement le juge des enfants en France. La loi de 1912 introduit donc du droit nouveau en France, mais elle marque moins une rupture avec le droit antérieur que la législation d'autres pays. C'est probablement pour cette raison qu'elle ne marquera une clôture des réformes que pour un temps plus bref que dans les autres pays : on sentira le besoin de reprendre les changements dès l'époque de la deuxième guerre.

\subsection{Les lois des Pays-Bas de 1922}

La chronologie de l'adoption des lois des Pays-Bas differe quelque peu de celle des autres pays que vise notre étude. Les Pays-Bas adoptent tout d'abord, en 1901, trois lois de synthèse sur la protection de l'enfance (lois qui entreront en vigueur en 1905). Formellement issus du gouvernement, ces projets de loi n'en sont pas moins le fruit d'initiatives des réseaux de philanthropie libérale et chrétienne qui, s'inquiétant particulièrement de la situation des institutions de rééducation, jouent un rôle moteur dans leur adoption. Un rapport d'une société philanthropique avait conclu, en 1898, à des résultats peu encourageants obtenus par des internats de rééducation et nombre de parents retiraient leurs enfants des institutions avant terme. On estimait ne pouvoir contrer le phénomène qu'en introduisant le retrait de l'autorité parentale dans la loi, comme l'avait fait en France la loi du 24 juillet 1889. C'est donc ce à quoi pourvoit la plus importante de ces trois lois, qui concerne la protection des enfants négligés ou délaissés. Les deux autres lois contiennent des dispositions de droit pénal visant les mineurs et règlent diverses questions 
relatives à la mise en œuvre des lois relatives à l'enfance (incluant la réglementation de certaines institutions). Il n'y est toutefois question ni de tribunaux pour mineurs, ni de liberté surveillée : on est encore à un moment où le modèle américain semble relativement peu connu en Europe. Il reste que, même si le régime institué par ces lois instaure une distinction entre enfants en danger et enfants délinquants en prévoyant pour les deux groupes des internats différents, certaines institutions prendront en charge des mineurs des deux groupes, et on étendra aux institutions de l'État qui prennent en charge la grande majorité des enfants délinquants un régime pédagogique développé à l'intention des enfants négligés, atténuant ainsi la différence de traitement des deux catégories d'enfants.

Deux ans à peine se sont écoulés après la mise en vigueur de ces lois qu'un débat s'amorce sur l'opportunité d'introduire la probation (liberté surveillée), au sujet de laquelle l'Angleterre vient de légiférer. On en implante la pratique dans plusieurs grandes villes, dont Amsterdam, sans pour autant l'encadrer par une loi. Des débats s'ensuivent : ne devraiton pas légiférer sur ces pratiques qui semblent donner de bons résultats dans des pays tels que la Belgique et les États-Unis ?

Quant aux tribunaux pour mineurs, ils avaient déjà été réclamés, lors des débats entourant l'adoption des lois de 1901, par un député socialiste qui insistait sur l'inaptitude pédagogique des magistrats. Avec le temps, d'autres voix se font entendre, particulièrement chez des juristes qui tirent leur inspiration d'exemples étrangers tels que l'Angleterre et la Belgique. Ici encore, une pratique se développe à Amsterdam, par laquelle une des chambres de la cour s'occupera exclusivement des affaires de mineurs.

À la suite d'un rapport de l'Assistance éducative et de l'Assistance publique de La Haye recommandant un encadrement légal pour la probation, demande est faite en 1918 à la Ligue néerlandaise pour la protection de l'enfance de prendre l'initiative de préparer un projet de législation. Une commission mixte est formée à cette fin : elle comprend des membres des réseaux privés de la protection de l'enfance et des députés. Au départ l'intention était surtout centrée sur l'idée d'un juge pour enfants délinquants; la commission modifie cette institution pour la 
centrer sur la mesure de probation. Les propositions de la commission sont acceptées sans grands changements par le ministre de la Justice, qui présente le projet au Parlement en 1920.

Deux lois sont donc adoptées en 1922. La première institue la probation, une mesure civile qui est vue comme un moyen terme entre l'absence de mesure et la déchéance de l'autorité parentale. Aux yeux du ministre de la Justice, elle vise à prévenir cette déchéance aussi bien que les placements en institution. Tout en demeurant une mesure civile, la probation sera souvent employée en droit pénal comme mesure complémentaire à une condamnation.

La seconde loi institue un juge des enfants qui est si intimement lié à la probation qu'on appelle «mineurs du juge des enfants " ceux qui sont sous probation. Ce juge est le seul qui puisse imposer la probation. Par contre, on l'intègre à la formation collégiale qui a compétence pour imposer les autres mesures de protection de l'enfance (essentiellement le retrait de l'autorité parentale).

Donc, en introduisant la probation et le juge des enfants en 1922, les Pays-Bas complètent le cheminement législatif entrepris en 1901. On ne saurait donc parler de "loi synthèse" sans renvoyer à l'ensemble de ces lois. Comme ailleurs, le processus prend assise sur des pratiques qui se sont développées et sur des débats publics. Les réseaux privés de la protection de l'enfance y jouent un rôle décisif, participant à la préparation de lois dont ils avaient eux-mêmes réclamé l'adoption. L'institution du juge des enfants y prend une forme particulière, étant en quelque sorte soudée à la probation d'une manière plus intime encore que dans les autres pays. Quelques années après la France, la Belgique et le Canada, les Pays-Bas introduisent la justice des mineurs et la probation dans leurs lois, en leur donnant toutefois une coloration particulière.

\section{Conclusion}

L'émergence de tribunaux pour mineurs et de la liberté surveillée dans divers pays à compter du début du XXème siècle constitue une balise majeure dans le développement d'un statut particulier aux mineurs. Elle n'en est toutefois pas le premier point de repère, bien au contraire. Ce 
statut avait commencé à se développer au cours du XIXème siècle : en sont témoins la création d'institutions spécifiques aux enfants, certaines lois visant la protection d'enfants en danger, l'instauration de procédures judiciaires particulières ainsi que le développement de pratiques diverses qui, sans être encadrées par des lois, constituent des bancs d'essai qui ultérieurement inspireront les législateurs. Tout spécialement aux États-Unis, les procédures judiciaires visant à autoriser le placement d'enfants avaient donné naissance à un modèle de protection de l'enfance qui serait par la suite au fondement même de l'orientation des tribunaux pour mineurs. Peut-on cependant prendre pour acquis que ce modèle fut adopté par les autres pays qui, dans les années qui suivirent la mise sur pied du tribunal de Chicago en 1899, adoptèrent à leur tour des lois instaurant des tribunaux pour mineurs ? Pour reprendre notre question de départ, l'émergence presque simultanée de ces lois au tournant du siècle est-elle le fruit de simples coïncidences chronologiques ou reflète-t-elle l'existence de convergences ? L'examen que nous avons fait de la question pour quatre pays appelle une réponse nuancée.

Le modèle protecteur qui est sous-jacent à ces entreprises législatives est présent dans les débats entourant les lois dans les quatre pays. Les textes eux-mêmes n'en portent toutefois pas la marque de la même façon. Deux pays adhèrent de manière particulièrement patente à ce modèle. La Belgique, tout d'abord, qui adopte une loi dont le titre même, "loi sur la protection de l'enfance ", indique l'orientation. Le Canada également qui, par sa loi sur les jeunes délinquants, veut clairement étendre à ces derniers un type d'intervention déjà développé dans le cadre d'une législation provinciale sur la protection de l'enfance. À leur manière, les Pays-Bas manifestent un souci du même ordre lorsqu'on y étend aux institutions pour délinquants un modèle éducatif conçu dans des milieux institutionnels réservés aux enfants négligés. La France fait toutefois exception : clairement présent dans les débats et dans les projets initiaux, le modèle protecteur voit sa présence graduellement évacuée à mesure qu'avancent les débats. En ce sens, l'on ne saurait dire que le modèle américain de justice des mineurs ait été retenu partout également, bien au contraire. 
La prégnance du modèle protecteur se traduit dans diverses mesures. L'une d'elles est la déchéance de la puissance paternelle, adoptée en France en 1889, aux Pays-Bas en 1901 et en Belgique en 1912. ${ }^{(18)}$

On l'observe tout autant dans le choix des acteurs à qui on en confie l'application. Cette approche a inspiré la conception américaine du juge des enfants : le juge paternel, bienveillant, qui a à cœur les intérêts de l'enfant, qui sait l'écouter et lui parler. C'est un juge unique : un collège de magistrats ne saurait créer l'ambiance requise pour l'exercice de cette fonction. La Belgique et le Canada optent pour ce type de juge. En France, ceux dont les positions sont les plus inspirées de ce modèle américain font la même option, mais leurs propositions ne sont pas retenues. À l'issue des débats, les parlementaires privilégient une formule où, sans doute, les affaires des mineurs seront entendues séparément de celles des adultes, avec une publicité restreinte et une procédure particulière, mais la loi n'impose pas le juge unique, spécialiste des affaires de mineurs. Enfin, aux Pays-Bas, l'institution du juge des mineurs prend une forme tout à fait particulière, liée à la probation.

Quelque variante que l'on retienne pour les fonctions du juge, chacun des quatre pays légifere en matière de liberté surveillée. L'agent de probation ou délégué à la liberté surveillée est le bras droit du juge des mineurs : il enquête, visite le mineur et les parents dans la famille, exerce divers contrôles et exécute les ordres du tribunal. Il est le second personnage du tribunal pour mineurs des États-Unis, formant un couple indissociable avec le juge des enfants dont il doit partager l'approche protectrice. Il est l'un des acteurs grâce auxquels l'univers philanthropique est censé s'insérer dans la justice des mineurs.

Nous sommes donc très loin d'une simple coïncidence en ce qui concerne le contenu des lois et des débats entourant leur adoption. Des convergences apparaissent clairement, en partie liées à l'adhésion à une approche protectrice. Elles sont particulièrement évidentes entre la Belgique et le Canada, dont les points de référence sont largement (mais non exclusivement) américains. Elles existent de la même manière avec ceux qui, en France, font des propositions inspirées des mêmes vues, même si, après débats et amendements, ces propositions ne sont pas
(18) Si la Constitution

du pays ne lui permet pas d'en faire autant, le Parlement fédéral canadien trouve d'autres moyens d'atteindre le même objectif, notamment en criminalisant et en sanctionnant les comportements parentaux susceptibles de favoriser la délinquance de leurs enfants. 
retenues. On peut enfin les retrouver dans les préoccupations de fond qui orientent les formules adoptées aux Pays-Bas, même si diverses modalités d'application revêtent un caractère national.

Des convergences caractérisent également le processus de production des lois dans les quatre pays. Même si c'est à des degrés divers, des initiatives privées issues de réseaux de protection de l'enfance contribuent largement à leur conception et à leur adoption. C'est évidemment le cas au Canada où des acteurs extraparlementaires associés aux sociétés d'aide à l'enfance conçoivent initialement le projet de loi et réussissent à le faire adopter malgré les réticences du ministre de la Justice. Aux PaysBas, les réseaux philanthropiques sont étroitement associés à la préparation de la loi. En France et en Belgique, leur apport est significatif en raison de leurs pressions, de leurs prises de position et de leur contribution aux débats. La mobilisation de l'opinion ressort comme une stratégie à laquelle ils ont largement recours, particulièrement en France, au Canada et en Belgique. Conférences publiques, pétitions et congrès internationaux sont au nombre des moyens utilisés. Rencontres et réseaux internationaux jouent un rôle particulièrement important $:$ ils influencent et facilitent l'adoption d'un modèle protecteur en Belgique et au Canada, en plus d'amener divers promoteurs français à endosser et soutenir - mais sans succès - la même orientation. Il serait abusif d'affirmer que, sans les interventions décisives de ces réseaux extraparlementaires, la justice des mineurs n'aurait pas vu le jour dans ces quatre pays. Il est toutefois clair que les lois qui l'ont instaurée n'auraient été adoptées que plus tard.

L'adoption de ces lois mettra un terme pour plusieurs décennies aux changements législatifs importants en matière de protection de la jeunesse. La France qui, en 1912, avait été la plus réticente à rompre avec le modèle pénal, sera la première à réviser sa loi d'une manière significative, en 1945.

Les convergences ne sont donc pas totales. Elles n'en sont pas moins pour autant très présentes, laissant plus ou moins d'espace, selon les pays, à l'impact de logiques et d'enjeux nationaux propres. 


\section{Références bibliographiques}

CAMPIONI (C.), Enquête sur la criminalité infantile, Bruxelles, Commission royale des patronages, 1908.

Comité de défense des enfants traduits en justice dans l'arrondissement de Bruxelles, Manuel de la défense de l'enfant traduit en justice devant le tribunal correctionnel de Bruxelles, 2ème éd., Bruxelles, 1906.

DESSERTINe (Dominique), MARADAN (Bernard), Pratiques judiciaires de l'Assistance éducative (1889-1941), Lyon, Centre Pierre Léon, université Lumière-Lyon 2, 1991.

DUPONT-BOUCHAT (Marie-Sylvie), PETIT (Jacques-Guy), DEKKER (Jeroen), FECTEAU (Jean-Marie), PIERRE (Éric), SCHNAPPER (Bernard), TÉTARD (Françoise), TRÉPANIER (Jean), avec la collaboration de MÉNARD (Sylvie) et STRIMELLE (Véronique), Enfants corrigés, enfants protégés. Genèse de la protection de l'enfance en Belgique, en France, aux Pays-Bas et au Québec (1820-1914), Paris, Rapport de recherche présenté au ministère français de la Justice, 1995.

DUPONT-BOUCHAT (Marie-Sylvie), PIERRE (Éric), [éd.], avec FECTEAU (Jean-Marie), TRÉPANIER (Jean), PETIT (Jacques-Guy), SCHNAPPER (Bernard), DEKKER (Jeroen), et avec la collaboration de MÉNARD (Sylvie) et STRIMELLE (Véronique), Enfance et justice au XIXème siècle. Essais d'histoire comparée de la protection de l'enfance, 1820-1914, France, Belgique, Pays-Bas, Canada, Paris, Presses universitaires de France, 2001. EMPEY (Lamar T.), STAFFORD (Mark C.), American Delinquency : Its Meaning and Construction, 3ème éd., Belmont, CA, Wadsworth Publishing Company, 1991.

FOX (Sanford J.), " Juvenile justice reform : An historical perspective ", Stanford Law Review, n 22, p. 1187-1239.

HAGAN (J.), LEON (J.S.), " Rediscovering delinquency : social history, political ideology and the sociology of law ». American Sociological Review, n 42, 1977, p. 587-598.

LABERGE (Danielle), Marginaux et marginalité : Les États-Unis aux XVIIème et XIXème siècles, Paris, L'Harmattan, 1997. 
NYQUIST (Ola), Juvenile Justice, Londres, Macmillan (Cambridge Studies in Criminology, vol. 12), 1960.

PLATT (Anthony M.), The Child Savers : The Invention of Delinquency, 2ème éd., Chicago, University of Chicago Press, 1977.

ROBERT (Philippe), Traité de droit des mineurs, Paris, éditions Cujas, 1969.

SCHLOSSMAn (Steven L.), Love and the American Delinquent: The Theory and Practice of "Progressive" Juvenile Justice, 1825-1920, Chicago, University of Chicago Press, 1977.

SCHLOSSMAN (Steven L.), "Delinquent children : The juvenile reform school ", in Morris Norval et David J. Rothman, [éd.], The Oxford History of the Prison: The Practice of Punishment in Western Society, p. 363389, Oxford, Oxford University Press, 1995.

SEYMOUR (John), Dealing With Young Offenders, North Ride, N.S.W., The Law Book Co, 1998.

VENTRELL (Marvin) (1998). " Evolution of the dependency component of the juvenile court ", Juvenile and Family Court Journal, $n^{\circ}$ 49/4, p. 17-37. 